\title{
10 Ein Fazit
}

Ganz verschiedene Gründe konnten dazu führen, sich als Südosteuropäer einer der vier Waffen-SS-Divisionen anzuschließen. Zumeist war es eine vielfältige Mischung aus Zwängen und der Hoffnung, durch eine Teilnahme am Krieg auf Seiten der Deutschen, das Leben für sich und seine Angehörigen angenehmer $\mathrm{zu}$ gestalten. Gleichzeitig bestanden auch auf Seiten der deutschen Besatzer zahlreiche Handlungszwänge, denen sie im Laufe der vier Jahre, 1941-1944, ausgesetzt waren oder die sie selbst mitverantworteten, indem sie etwa bereits schwelende lokale interethnische Konflikte noch verschärften. Auch die Dynamik zwischen erlebter und ausgeübter Gewalt an der Ostfront bzw. während des Dienstes in Konzentrationslagern und den lokalen Gewaltvorkommen ist hierzu zu zählen: Die Überlappung verschiedener Konflikte, einerseits auf internationaler Ebene der Zweite Weltkrieg, andererseits auf lokaler Ebene verschiedene Bürgerkriege, die in Südosteuropa ausgefochten wurden, kurbelte die Spirale der Gewalt zusätzlich an. Dabei trafen bereits brutalisiserte deutsche und „volskdeutsche“ Vorgesetzte auf bereits brutalisierte südosteuropäische Soldaten. Wie gezeigt wurde die überbordende Gewalt an Zivilisten zum Teil auf Befehl ausgeführt, teilweise aber auch aufgrund individueller Entscheidungen auf Mannschaftsebene.

Ist Südosteuropa auch wegen der Rekrutierungen für die verschiedenen Formationen der Waffen-SS eine konfliktreiche Region geblieben? - Dies habe ich mich in den letzten fünf Jahren oft gefragt. In gewisser Weise ist diese Frage mit ,ja“ zu beantworten, auch wenn es dabei zahlreiche Einschränkungen gibt. Nicht unbedingt die Rekrutierungen selbst oder die begangenen Kriegsverbrechen, sondern das Verbot, darüber zu sprechen und nach Motivationshintergründen der beteiligten Individuen oder Gruppen zu fragen, halfen, dem Mythos „Waffen-SS als Motor zur Durchsetzung nationaler Interessen“ Vorschub zu leisten. Die Verbannung der Erinnerung an Angehörige der „Handschar“, „Skanderbeg“, „Kama“ und „Prinz Eugen“ in den privaten Raum ermöglichte ein unreflektiertes Weitererzählen der deutschen Rekrutierungsvorgänge in Südosteuropa und der Taten der Divisionsangehörigen. Heute wird in Ost- und Südosteuropa der Dienst in einer dieser Waffen-SS-Divisionen vielfach als Dienst an der nationalen Sache gesehen und ehemalige Divisionsangehörige posthum ausgezeichnet. ${ }^{1}$ Was die Waffen-SS eigentlich war und wofür sie stand, wird dabei ausgeblendet.

Doch drehen wir das Rad der Zeit zurück, um zu erklären, weshalb zahlreiche Vorgänge ungeklärt blieben und schließlich totgeschwiegen wurden: Im Jahre 1963 kommt in Deutschland ein angetrunkener Mann auf eine Polizeiwa-

\footnotetext{
1 Bild der Auszeichnung ist in Besitz der Autorin.
}

Ә Open Access. (c) 2021 Franziska Anna Zaugg, publiziert von De Gruyter. (cc)BY Dieses Werk ist lizenziert unter einer Creative Commons Namensnennung 4.0 International Lizenz. 
che in Osnabrück. Er möchte ein Geständnis ablegen. Er gibt an, als Kommandeur einer kleinen Einheit der Division „Handschar“ Massaker in drei Ortschaften befohlen zu haben. Günter Borkowsky sucht einen Umgang mit der Vergangenheit; mit seiner Vergangenheit. Nach knapp zwanzig Jahren will er nicht mehr schweigen. Die Leserin bzw. der Leser der Akten denkt, dass dies seine Richtigkeit habe: Der Täter stellt sich den Behörden und sich selbst.

Die Reaktion der Behörden weist jedoch in eine gegenteilige Richtung: Der Fall wird nach etlichen Zeugenaussagen, die teilweise widersprüchlich sind, eingestellt. Für die Bundesrepublik Deutschland der 1960er Jahre stellte ein solches behördliches Verhalten nicht die Ausnahme, sondern die Regel dar. ${ }^{2}$ Obwohl 1958 die „Zentrale Stelle der Landesjustizverwaltungen zur Aufklärung nationalsozialistischer Verbrechen“ ihre Arbeit aufnahm und Anfang der 1960er Jahre mit neuen Anklagen, Verhaftungen und Verhören eine Systematisierung der Strafverfolgung einsetzte, wurde dieses Vorgehen weder von der deutschen Verwaltung noch von der Mehrheit der deutschen Bevölkerung gutgeheißen. Man hatte sich auf eine Vergangenheitsbewältigung geeinigt, die gleichermaßen auf Amnestie und Amnesie gründete. ${ }^{3}$

Im Raum bleiben Orte wie Čelić, Brčko, Vinkovci, wo Verbrechen stattgefunden haben; und in diesen Fällen hatte wahrscheinlich Borkowsky den Befehl dazu gegeben. Bei der Bevölkerung vor Ort, in Jugoslawien, wird nicht nachgefragt; sie hätte über die Erschießungen und Massengräber vielleicht wichtige Hinweise liefern können. Das Vorgehen in Jugoslawien unter Tito weist nach drei Phasen der Verfolgung, die kurz nach dem Krieg auf spontaner Gewalt und später auf Gerichtsprozessen basierten, Ähnlichkeiten mit demjenigen in Deutschland der frühen 1960er Jahre auf: Tito verordnete eine kollektive Amnesie.

Rekrutierungen für verschiedenste Formationen des rechten Spektrums von der Ustaša bis zur Waffen-SS und extrem gewalttätige Konflikte zwischen diesen und den Partisaneneinheiten, sowie zwischen Angehörigen verschiedener ethnischer Gruppen während des Zweiten Weltkriegs, sollten mit verordnetem Vergessen überwunden werden. Letztlich führte das Nicht-Sprechen-Können über die Vergangenheit zu einem übersteigerten Nationalismus, welcher der Föderativen Volksrepublik Jugoslawien ein Ende setzte und dessen Wortführer die einzelnen Bevölkerungsgruppen wieder gegeneinander hetzten. ${ }^{4}$

2 Vgl. Orkoy, Mammutprozess gegen Polizisten, in: Schulte, SS, Himmler und die Wewelsburg, S. 449-469.

3 Vgl. ebd., S. 455. Vgl. zum Auf- bzw. Ausbau der HIAG im Spiegel der Systematisierung der Strafverfolgung Wilke, Die Truppenkameradschaften der Waffen-SS, S. 425.

4 Calic bezeichnet etwa die Mythenbildung um Bleiburg und die foibe (Karstschluchten/höhlen) als Zeitbomben. Vgl. Calic, Geschichte Jugoslawiens, S. 173. 
So sprach etwa Slobodan Milošević in seiner berühmten Rede auf dem Amselfeld (Kosovo Polje) vom 28. Juni 1989: „Die fehlende Einheit und der Verrat im Kosovo sollten das serbische Volk wie ein grausames Schicksal durch seine gesamte Geschichte verfolgen. Sogar im letzten Krieg [Zweiter Weltkrieg] führten sie das serbische Volk und Serbien in ein grausames Schicksal, dessen Folgen historisch und moralisch die der faschistischen Aggression überstiegen. " ${ }^{\text {" Und er }}$ meinte damit den Verrat durch diejenigen Männer, die mit den Deutschen zusammengearbeitet hatten und sich für ihre Waffen-SS, Wehrmacht und Polizei hatten rekrutieren lassen. Dieser Verrat durch die „eigenen“ Leute, so Milošević, wog schwerer als die Gewalt der Deutschen. ${ }^{6}$

Was blieb waren Wunden, die im ideologisierten Geschichtsbewusstsein des real existierenden Sozialismus unter Tito und Enver Hoxha nicht geheilt werden konnten. ${ }^{7}$ Wie spätestens die Konflikte der 1990er Jahre, in welchen Versatzstücke alter Uniformen auch die neuen Gegner kennzeichneten, demonstriert haben, war Titos Strategie gescheitert: Eine nicht bewältigte Vergangenheit wurde abermals nationalistisch aufgeladen und instrumentalisiert. ${ }^{8}$ Insbesondere Regionen wie Kosovo und Ostbosnien wurden erneut von heftigen Kämpfen heimgesucht. Viele Orte, die bereits im Zweiten Weltkrieg von Gewaltexzessen betroffen waren, wurden in den 1990er Jahren wieder zum Schauplatz von Gewalt. ${ }^{9}$

Folgt man den Spuren der südosteuropäischen Waffen-SS-Divisionen bis heute, kann man einen Zusammenhang zwischen der Unterdrückung von Reden über Vergangenheit und ihrer Monopolisierung durch die jugoslawischen, albanischen oder rumänischen Sozialisten und der damit einhergehende Privatisierung von Überlieferung feststellen: Obwohl in der Öffentlichkeit mit einer Damnatio Memoriae belegt, lebten die Taten der ehemaligen Waffen-SS-Angehörigen aus Bosnien, Kosovo, Serbien, Rumänien und weiteren Staaten im Familienkreis fort. In Vereinen organisiert, wobei die HIAG die wohl bekannteste Organisation ist, versuchten gleichzeitig auch in Deutschland und Österreich ehemalige Waffen-SSAngehörige ihre Taten in apologetischen Schriften $\mathrm{zu}$ beschönigen. ${ }^{10}$

Dass nationalistischen Kräften und einer apologietrunkenen, rechtsgerichteten Veteranenminderheit über Jahrzehnte die Aufarbeitung der Geschichte der Re-

5 http://wwwg.uni-klu.ac.at/eeo/Milosevic_Rede (Stand: 8.8.2018), S. 649.

6 Ebd.

7 Siehe zum verodrneten Schweigen Ferhadbegović, Vor Gericht, S. 250.

8 Vgl. ebd., S. 250.

9 Vgl. Etwa Robert Hayden, Mass Killings and Images of Genocide in Bosnia, 1941-1945 and 1992-1995, S. 137-169.

10 Etwa Nation Europa, Monatsschrift im Dienst der europäischen Erneuerung, BArchF, N 756/149a. 
krutierungen von Südosteuropäern in Waffen-SS-Divisionen überlassen wurde, stimmt nachdenklich. Das in den letzten zehn Jahren zunehmend breitere Forschungsinteresse an Rekrutierungen von Waffen-SS-Soldaten aus Südosteuropa soll mit dieser Studie auf eine neue Stufe gehoben werden; indem die vier südosteuropäischen Divisionen erstmals gemeinsam betrachtet, untersucht und verglichen wurden. Es ging dabei insbesondere um die Einbettung der Rekrutierungen in einen breiteren kulturhistorischen Kontext, der es erlaubte, die Wechselwirkungen zwischen deutschen Besatzern, nationalen Regierungen, lokalen Eliten und einfachen Zivilisten aufzuzeigen und die Handlungsmacht, die Motivation als auch die Zwänge der einzelnen Gruppen und Individuen zu erklären. 